\title{
DEVELOPMENT OF FROVATRIPTAN SUCCINATE MICROEMULSION FOR NASAL DELIVERY: OPTIMIZATION, IN VITRO AND IN VIVO EVALUATION
}

\author{
UPENDRA C GALGATTE ${ }^{1,2 *}$, PRAVIN D CHAUDHARI ${ }^{1}$ \\ ${ }^{1}$ Department of Pharmaceutics, Modern College of Pharmacy, Sector No. 21, Yamunanagar, Nigdi, Pune, India. ${ }^{2}$ Jawaharlal Nehru \\ Technological University, Hyderabad, Telangana, India. Email: ucgpharm@rediffmail.com
}

Received: 03 January 2019, Revised and Accepted: 18 March 2019

\section{ABSTRACT}

Objective: The main objective of the present research work was to develop, optimize, and characterize microemulsion (ME) of frovatriptan succinate to improve brain transport.

Methods: The pseudoternary phase diagrams were constructed for ME formulations composed of Capmul MCM, Cremophor EL, and propylene glycol. Frovatriptan succinate-loaded ME was optimized by simplex lattice design having the concentration of oil, surfactant, and cosurfactant representing three apex points on the triangle. These were taken as independent variables and percentage drug release as a response variable. All developed batches of ME were characterized for in vitro tests, histopathology study, and pharmacokinetics in Swiss albino rats.

Results: Clear MEs were obtained. F5 having particle size $142.0 \mathrm{~nm}$, zeta potential -17.7 to $-7.8 \mathrm{mv}$, refractive index $1.37 \pm 0.21$, drug content $98.25 \pm 1.10 \%$, and drug diffused through dialysis membrane $85 \%$ was was the optimized batch. Drug permeation through the nasal mucosa of F5 in the ex vivo study was found to be $82.32 \%$. Histopathology microscopic study has shown that F5 does not cause any irritation and structural changes in sheep nasal mucosa. The pharmacokinetic parameters were determined after nasal and oral administration of F5. For brain tissue, after nasal administration were $\mathrm{C}_{\max } 181 \pm 1.51 \mathrm{ng} / \mathrm{ml}, \mathrm{T}_{\max } 2 \pm 1.01$, area under curve (AUC) $)_{0-6} 390.0 \pm 2.08 \mathrm{ng}$.h $/ \mathrm{ml}_{\text {. The AUC }}$ attained by nasal ME was 3.29 times greater than oral solution. Drug targeting index of frovatriptan succinate was 2.06. This was found satisfactory.

Conclusion: Microemulsion of said composition was found to be enhancing delivery of frovatriptan succinate to brain tissues through nasal route.

Keywords: Drug targeting index, Frovatriptan, Liquid chromatography-mass spectrometry, Microemulsion, Nasal.

(C) 2019 The Authors. Published by Innovare Academic Sciences Pvt Ltd. This is an open access article under the CC BY license (http://creativecommons. org/licenses/by/4. 0/) DOI: http://dx.doi.org/10.22159/ajpcr.2019.v12i4.31830

\section{INTRODUCTION}

The nasal mucosa has been considered as a route of administration to speed up a higher level of drug absorption. Among the mucosal sites in the human body, nasal mucosa has the potential to compete with the intravenous route for fast onset of action and percentage bioavailability [1]. This potential lies in the ability of nasal mucosa of high drug permeation due to exceedingly vascularization. In addition, the olfactory region of the nose has the potential to stream a drug to the central nervous system (CNS) [2]. Therefore, this offers the possibility to target CNS by bypassing blood-brain barrier (BBB) [3]. The drugs absorbed nasally through olfactory epithelium enter in olfactory neurons and supporting cells and subsequently into the brain, which reduced the systemic toxicity of centrally acting drugs and enhanced therapeutic efficacy [4]. Rapid onset of action is attributed by high permeability of nasal mucosa with large surface area [5]. In addition to this, nasal administration minimizes the lag time associated with oral drug delivery and offers non-invasiveness [6].

Microemulsion is a transparent looking product having a droplet size of 20-200 nm composing of oil, surfactant, cosurfactant, and water at suitable proportions. It has several specific physicochemical properties such as transparency, optical isotropy, low viscosity, and thermodynamic stability [7]. These properties made it possible to use in a drug delivery system. Hydrophilic and lipophilic drugs can be administered through microemulsion (ME) due to its oil and aqueous composition. Drug dissolved in components of the ME can cross the BBB due to its lipophilicity. Hence, ultimately, it is promising for nasal delivery of drugs. There are pieces of evidence of ME used as intranasal drug delivery systems targeting to the brain.
Migraine is a neurological disorder with intense, unilateral, throbbing, and pulsatile headache attacks that may last for $4-72 \mathrm{~h}$ and may be accompanied by anorexia, nausea, vomiting, photophobia, and/or phonophobia [8]. In connection with migraine, drugs used in treatment must be absorbed immediately for fast onset of action and high bioavailability. This need can be successfully fulfilled by administration of drug-loaded ME through nasal route.

Frovatriptan succinate has a uniquely long-half life than that of other triptans. This provides the opportunity to use frovatriptan in menstrual-related migraine and other situations, as well as use in long-lasting or recurrent migraine. In the pathogenesis of migraine, neurogenic inflammation and cerebral vasodilatation are significant factors. Activation of $5-\mathrm{HT}_{1 \mathrm{D}}$ prevents neurogenic inflammation, and activation of $5-\mathrm{HT}_{1 \mathrm{~B}}$ reverses cerebral vasodilatation. Frovatriptan has a high affinity for 5- $\mathrm{HT}_{1 \mathrm{D}}$ and 5- $\mathrm{HT}_{1 \mathrm{~B}}$ receptors. Nonetheless, frovatriptan has a moderate affinity for $5-\mathrm{HT}_{1 \mathrm{~A}}$ and $5-\mathrm{HT}_{1 \mathrm{~F}}$ receptors subtypes, and it is most potent $5-\mathrm{HT}_{1 \mathrm{~B}}$ agonists [9]. Incomplete absorption from the gastrointestinal tract is attributing to the low oral bioavailability of frovatriptan that is $22 \%-30 \%$ [10]. Although a single dose 2.5 $\mathrm{mg}$ is recommended, additional doses up to $7.5 \mathrm{mg}$ in a day may be administered with intervals of more than $2 \mathrm{~h}$ on the basis of recurrence of headache. Animal studies have proven that frovatriptan has limited capacity to cross the BBB [11].

As intranasal administration offers a practical, non-invasive route of administration for drug delivery to the brain $[12,13]$ and intranasal microemulsions are promising carriers for achieving the goals in drug targeting to brain $[14,15]$; the objective of this study was to formulate and optimize microemulsion of frovatriptan succinate for intranasal 
delivery. In this work, frovatriptan succinate loaded microemulsions were designed by using a simplex lattice experimental design and characterized. Microemulsion formulation was optimized and further evaluated for drug targeting index (DTI).

\section{MATERIALS AND METHODS}

\section{Materials}

Frovatriptan succinate was obtained as a gift sample from Glenmark Generic Ltd., Navi Mumbai, India. Capmul MCM was obtained as a gift sample from Abitec Corporation. Cremophor EL and Cremophor RH 40 were obtained as a gift sample from BASF India. Labrafil M 2125 CS, Maisine 35-1, and Plurol Oleique were procured from Gattefosse India Pvt. Ltd., Mumbai, India. Tween 80, propylene glycol, and PEG 400 were purchased from Molychem, India. Hariol 538 mediumchain triglycerides (MCT oil) and Labrasol were gifted from Subhash Chemical Industries Pvt. Ltd., Pune, India. All other ingredients used were of analytical grade.

\section{Methods}

Estimation of drug content in oils, surfactant, and cosurfactant: Oils, surfactants, and cosurfactant were tested for solubility of frovatriptan. A suitable solvent system was developed to estimate drug solubility in selected ME components. Combination of phosphate buffer $\mathrm{pH} 6.4$ and methanol found appropriate one. Ratios such as 1:9, 2:8, 3:7, 4:6, $5: 5,6: 4,7: 3,8: 2$, and 9:1 v/v were prepared and used as solvent for extraction and estimation of drug from ME components. The selected ratio was optimized on the basis of solubility of ME component in it. Further, the same solvent combination was used to develop calibration curve. Different dilutions of concentrations $2-10 \mu \mathrm{g} / \mathrm{ml}$ were prepared and absorbances $(n=3)$ of all dilutions were noted at fixed $\lambda_{\max } 244 \mathrm{~nm}$ using Ultraviolet (UV) Spectrophotometer (Shimadzu-1800).

\section{Solubility studies}

Capmul MCM, oleic acid, isopropyl myristate, Maisine 35-1, and Hariol 538 MCT were selected as oil component for solubility testing. Cremophor EL, cremophor RH-40, tween 80, Labrafil M 2125 CS, and Labrasol were surfactants and propylene glycol, plurol oleique, PEG 400, and ethanol were tested as surfactant and cosurfactant, respectively. To define supersaturation solubility, an excess amount of drug was added in each vial holding $2 \mathrm{ml}$ of above emulsion components separately [16]. Each vial was ultrasonicated to dissolve the excess amount of drug up to supersaturation. The vials were kept in orbital shaker for $48 \mathrm{~h}$ with stirring. Samples were centrifuged at $7500 \mathrm{rpm}$ for $15 \mathrm{~min}$ to obtain a sufficient amount of supernatant. The drug in this supernatant was analyzed by making proper dilution with an optimized ratio of solvent by UV spectrophotometer at $244 \mathrm{~nm}$ to estimate the solubility of drug.

Construction of phase diagram and preparation of ME formulation To construct pseudoternary phase diagrams, surfactant:cosurfactant ratio was termed as $\mathrm{S}_{\text {mix }}$ and considered it for one apex of a triangle. Rest of the two apexes were of oil and water. A water titration method was found suitable to develop different phase diagrams [17]. $S_{\text {mix }}$ was Cremophor EL: Propylene glycol; 2:1, 3:1, and 4:1 w/w. Selected S was blended with oil in weight ratios of 1:9, 2:8, 3:7, 4:6, 5:5, 6:4, 7:3, $8: 2$, and $9: 1$. Water was added drop by drop to each mixture under proper magnetic stirring at $37^{\circ} \mathrm{C}$ until the mixture became clear at a certain point. After equilibrium, the samples were visually checked and determined as being clear MEs [18]. Chemix software (free version) was right and proper to create pseudoternary phase diagrams. After the identification of ME region in the phase diagrams, the contents of oil, $\mathrm{S}_{\text {mix }}$ and water at appropriate weight ratios were selected. The MEs were selected at desired component ratios. The preparation of selected ME was simply performed by adding the weighed components together and stirring to form the clear ME.

\section{Preparation of drug-loaded ME}

According to the ME formation area in the phase diagrams, the frovatriptan succinate-loaded ME formulations were selected at different component ratios. Frovatriptan succinate was added to the mixtures of oil, surfactant, and cosurfactant under gentle ultrasonication with varying component ratios. Then, an appropriate amount of distilled water was added to the mixture drop by drop and the ME containing frovatriptan succinate was obtained by stirring the mixtures at room temperature at constant rpm.

Formulation and optimization of frovatriptan succinate-loaded ME The simplex lattice design for a three-component system consists of an equilateral triangle in two-dimensional space [19]. Seven batches (F1-F7) were prepared by taking three independent variables; one at each vertex $\left(\mathrm{X}_{1}, \mathrm{X}_{2}\right.$, and $\left.\mathrm{X}_{3}\right)$, one at the halfway point between vertices $\left(\mathrm{X}_{1} \mathrm{X}_{2}, \mathrm{X}_{2} \mathrm{X}_{3}\right.$, and $\left.\mathrm{X}_{1} \mathrm{X}_{3}\right)$, and one at the center point $\left(\mathrm{X}_{1} \mathrm{X}_{2} \mathrm{X}_{3}\right)$.

The concentration of oil, surfactant, and cosurfactant was selected as independent variables. The percentage of drug release after $7 \mathrm{~h}$ was taken as response.

A simplex lattice design was used using Design-Expert software (trial version). In this design, three factors were evaluated by changing their concentrations simultaneously and keeping their total concentration constant.

\section{Characterization of ME formulation}

Frovatriptan succinate-loaded MEs were characterized for appearance, $\mathrm{pH}$, viscosity, refractive index, in vitro drug diffusion study, particle size/globule size, zeta potential, and drug content.

\section{Identification: Type of ME}

It is tested for the type of MEs whether it is o/w or w/o type. A drop was put on a filter paper and observed for its spreading and absorption on filter paper [20].

pH of $M E$

The $\mathrm{pH}$ measurement for all MEs was carried out using digital $\mathrm{pH}$ meter.

\section{Viscosity}

Rheological behavior of MEs was noted at room temperature using small volume adapter of Brookfield Viscometer (Brookfield, RV DV II + Pro).

\section{Refractive index}

The refractive index of all MEs was determined using Abbe's refractometer

\section{In vitro drug diffusion study}

In vitro drug diffusion study of all formulations (F1-F7) was carried out using Franz diffusion cell [21] having the capacity of $17 \mathrm{ml}$ and dialysis membrane (HiMedia; molecular weight cutoff range 12000-14000 kDa) as diffusion membrane. Pre-diffusion soaking of dialysis membrane was carried out in phosphate buffer $\mathrm{pH} 6.4$ for 24 h. Diffusion cell was filled with phosphate buffer pH 6.4 and dialysis membrane was mounted on it. The temperature was maintained at $34 \pm 1^{\circ} \mathrm{C}$ by circulating water bath. The content of the receptor fluid was stirred continuously using a magnetic stirrer. Samples were withdrawn at different time intervals and replaced with the same volume of fresh sample. Samples were filtered and the amount of drug was estimated by UV-visible spectrophotometer at $244 \mathrm{~nm}$.

\section{Particle size and zeta potential}

Particle size of all formulations was measured using Zetasizer (Nanozydus 90 Malvern Ltd., U.K.). All samples were diluted in a 1:10 by volume with deionized water to get optimum 100-200 Kilo counts/s (KCPS) for measurements.

\section{Drug content}

A sample of $0.1 \mathrm{ml}$ of ME was added in selected solvent ratio: Phosphate buffers $\mathrm{pH}$ 6.4:methanol (1:9v/v). Methanol of high-performance liquid chromatography grade used was filtered, and then, the volume was 
made up to $10 \mathrm{ml}$. A $1 \mathrm{ml}$ of this solution was successfully diluted 100 folds. The resultant solution was analyzed by UV spectrophotometer at $244 \mathrm{~nm}$. Drug-free ME (blank ME) was also diluted in a similar manner and detected at $244 \mathrm{~nm}$ [22]. Frovatriptan content was extrapolated from the standard curve.

\section{Ex vivo permeation study}

Ex vivo permeation study was carried out on sheep nasal mucosa. A fresh nasal mucosa was collected from a local slaughter house. Mucosa of the olfactory region was targeted to separate skillfully. After separating mucosa carefully from nasal tissues, it was kept in phosphate buffer $\mathrm{pH} 6.4$ for $1 \mathrm{~h}$ to obtain good permeation of drug after saturation with phosphate buffer [23]. Thickness of nasal mucosa was 0.85-0.95 mm; exposed area of mucosa on diffusion cell membrane was approximately $2.98 \mathrm{~cm}^{2}$. Ex vivo diffusion drug release was carried out using 6 station diffusion cell apparatus (Make: Orchid). At different time intervals, sample was withdrawn, then filtered through Whatman filter paper $(0.45 \mu \mathrm{m})$, and analyzed using UV spectrophotometer. Experimental conditions such as temperature, the volume of replacement were the same like in vitro drug release study.

\section{Histopathological studies}

Fresh nasal mucosa used in ex vivo permeation study was carefully removed from diffusion cell and stored in $10 \%$ of formalin solution to avoid bacterial growth. Further, mucosa was cut and stained with eosin. One portion of mucosa was treated with phosphate buffer $\mathrm{pH}$ 6.4 and other with isopropyl alcohol (IPA). These samples were used as a negative and positive control, respectively. All three sections were examined under $40 \times$ by light microscope (Motic DMW, $\mathrm{B}_{1}-223 \mathrm{ASC}$, Motic Incorporation Pvt. Ltd.) for microscopic appearance and structural alterations if any.

Mucoadhesive strength of the optimized formulation was carried out using a modification of the mucoadhesive strength measuring device used by Choi et al. [24]. A fresh sheep nasal mucosa was put between the two glass vials with ME applied at one side, and the force required to detach the vials was measured.

\section{Animal study}

The protocol of the animal study was approved by the Institutional Animal Ethical Committee of Modern College of Pharmacy, Nigdi, Pune, having reference No: MCP/IAEC/35/2011 as per CPCSEA guidelines. Swiss albino rats weighing 200-250 g were used for the study. These were obtained from the National Toxicological Centre, Pune, India.

\section{Analytical procedure}

Liquid chromatography-mass spectrometry (LC/MS) technique was developed for analysis of drug using C-18, $150 \times 4.6 \mathrm{~mm}, 5 \mu \mathrm{m}$ (Chromatopack). For plasma and brain tissue samples, the mobile phase consists of $0.1 \%$ formic acid in methanol (organic):ammonium acetate buffer $10 \mathrm{Mm}$ (aqueous) (85:15) v/v was used. The flow rate was $1.0 \mathrm{ml} / \mathrm{min}$ at ambient temperature. Detection was performed using an API-4000 QTRAP instrument (Applied Biosciences AB Sciex) [25].

\section{Sample preparation}

The microemulsion was weighed accurately equivalent to $5.0 \mathrm{mg}$ of frovatriptan succinate and dissolved in methanol:water. Volume adjusted up to the mark $5.0 \mathrm{ml}$. Thus, $5 \mathrm{mg} / 5 \mathrm{ml}$ main stock $(1000$ ppm) was prepared. Further, dilutions were made in the range of 10 , $20,50,200,400,600$, and $800 \mathrm{ng} / \mathrm{ml}$. Metoclopramide hydrochloride $1 \mathrm{ng} / \mathrm{ml}$ was used as an internal standard. Retention time was 2.08 min for frovatriptan succinate and $2.18 \mathrm{~min}$ for internal standard metoclopramide hydrochloride.

\section{Animal experiment and drug administration}

A $12 \mathrm{~h}$ before and during the pharmacokinetic study, Swiss albino rats were fasted. However, they were allowed free access to water. The animals were divided into two: Groups A and B in such a way that, to one group, nasal ME formulation was administered (Group A) and, to another group, oral aqueous formulation of frovatriptan succinate (Group B) was administered. Each group consists of six rats. The blood samples and brain tissue were collected from each group to analyze the quantity of drug after administration by both routes. For Group A, optimized ME formulation was administered nasally, and for Group B, aqueous solution was administered.

\section{Sample collection and analysis}

After administration, blood samples from each group were withdrawn from the retro-orbital vein at time intervals of 0.16 (10 $\mathrm{min}), 2,4,5$, and $6 \mathrm{~h}$ and were collected in EDTA tubes to prevent clotting of blood. Blood samples were centrifuged at $5000 \mathrm{rpm}$ for $10 \mathrm{~min}$ to separate plasma. Animals were sacrificed for collection of brain tissue at time intervals of 0.16 (10 $\mathrm{min}), 2,4$, and $6 \mathrm{~h}$ from each group. Brain tissue was separated carefully and then rinsed with normal saline solution and kept in tissue paper to remove the excess of blood. Brain tissue was mixed with 1 volume normal saline solution and homogenated in a tissue homogenizer, and supernatant was collected for analysis. Plasma samples and brain tissue homogenates were stored at $-20^{\circ} \mathrm{C}$ until analysis. Drug concentrations in plasma samples and brain tissue homogenates were determined using LC/ MS technique. Internal standard (metoclopramide hydrochloride) $20 \mu \mathrm{l}$ was added. Plasma and brain tissue homogenates samples were extracted. A mixture of diethyl ether and dichloromethane, 50:50 parts, was used as extracting solvent. A $2 \mathrm{ml}$ of solvent was added and mixed and then centrifuged at $10,000 \mathrm{rpm}\left(8^{\circ}\right)$. Flash precipitation with chilled methanol took place. The supernatant was decanted thereafter. The remaining contents were evaporated at $35^{\circ} \mathrm{C}$ with the help of nitrogen evaporator. A mobile phase $200 \mu \mathrm{l}$ was used to reconstitute the samples for LC/MS analysis. After extraction, study samples were run. These samples were run along with the calibration curve for quantitative analysis.

\section{Pharmacokinetic studies}

Pharmacokinetic parameters were derived from the study samples versus time plot. AUC, $\mathrm{C}_{\text {max }}$ and $\mathrm{T}_{\max }$ were estimated. AUC was statistically analyzed using software GraphPad Prism version 5.01. Brain targeting of drug was calculated by DTI as given by the following equation [26].

$$
\text { DTI }=\frac{\text { (AUC brain tissue } / \text { AUC plasma)i.n. }}{\text { (AUC brain tissue / AUC plasma)oral }}
$$

\section{RESULTS AND DISCUSSION}

Estimation of drug content in oils, surfactants, and cosurfactants The mixture of phosphate buffer $\mathrm{pH} 6.4$ and methanol 1:9 v/v was selected to solubilize the drug from ME components, i.e. oil, surfactant, and cosurfactant. Various proportions of phosphate buffer $\mathrm{pH} 6.4$ and methanol 9:1v/v-1:9 v/v were evaluated on the basis of solubility of a drug from ME components. The turbidity was observed where the proportion of phosphate buffer $\mathrm{pH} 6.4$ was more. However, clarity improved as the proportion of methanol increased. Phosphate buffer pH6.4 and methanol in the ratio $1: 9 \mathrm{v} / \mathrm{v}$ showed more clarity after miscibility of ME components in it, and hence, it was selected. This selected ratio of solvent was used to develop calibration curve of drug in it. $\lambda_{\max }$ was found to be $244 \mathrm{~nm}$ (Linearity was observed from 2 to $10 \mu \mathrm{g} / \mathrm{ml}$, and $\mathrm{R}^{2}$ was 0.999 ).

\section{Solubility studies}

Oils of semi-synthetic nature were included in the present study. These were preferred intentionally to obtain reproducibility in results and stability of preparation. The order of solubility of frovatriptan succinate among the oils screened was capmul MCM followed by isopropyl myristate and oleic acid.

The oil components constitute significant proportion in a ME formulation and the solubility in Capmul MCM was found and surfactant together is more than water alone. This value signifies drug carrying capacity and possible delivery to the brain. In practice, the drug gets solubilize in all components of a ME. Therefore, this affects drug loading, clarity, and overall appearance of formulation. Therefore, it was relevant to screen 
surfactants and cosurfactants for solubility of the drug. Surfactants such as Cremophor EL showed maximum solubility of frovatriptan succinate followed by Tween 80 . Cosurfactants such as propylene glycol and ethanol also showed good solubility of frovatriptan succinate in it. Solubility study was carried out at temperature $37 \pm 2^{\circ} \mathrm{C}$. Excipients showing maximum solubility were used for the phase behavior study. As more drug loading was possible with this selection, Capmul MCM as oil phase, Cremophor EL as surfactant, and propylene glycol as cosurfactant were selected for further study. These components showed maximum solubility than other so selected for development of the ME formulation. It was thought that, apart from the role of surfactant and cosurfactant to reduce interfacial tension between oil and water components and to make them more miscible into each other, they are also serving as a solvent for frovatriptan succinate. Practically, it was observed that surfactants and cosurfactants have shown considerable solvent property for solubilization of frovatriptan succinate. Various oils, surfactants, and cosurfactants used for solubility studies are listed in Table 1. Oils, surfactants, and cosurfactants showing maximum solubility of drug were selected for further studies.

Construction of phase diagram and preparation of ME formulation Pseudoternary phase diagrams consisting of oil (Capmul MCM), surfactant (Cremophor EL), cosurfactant (propylene glycol), and water are shown in Fig. 1, from which exact concentration ranges of different components of ME composition were obtained. The ME regions of $\mathrm{S}_{\text {mix }}$ in various ratios 4:1, 3:1, and 2:1 were identified.
The shaded area in the diagrams shows the formation of the ME systems at room temperature. It was concluded that the highest ME zone was achieved for the MEs containing cremophor EL/propylene glycol at a ratio of 2:1. A maximum amount of water uptake was found for Capmul MCM and Cremophor EL/propylene glycol at 2:1 concentration [Table 2].

\section{Preparation of drug-loaded ME}

Frovatriptan succinate was partitioned into all components of the ME and finally formed a clear ME. Incorporation of frovatriptan succinate was found easy.

\section{Characterization of ME formulations \\ Identification: Dilution test}

No any phase separation was observed when diluted with an excess of water. However, when diluted with oil, immediately phase separation was observed. This indicated that continuous phase was water, and hence, ME was o/w type.

\section{Identification: Filter paper test}

A drop of ME was placed on a filter paper. Spreading and absorption of drop the surface indicated $\mathrm{o} / \mathrm{w}$ type of ME. This test was carried out to confirm the above test.

\section{$\mathrm{pH}$ of ME}

The $\mathrm{pH}$ values of ME formulation are shown in Table 3. The $\mathrm{pH}$ values of all the formulations were found compatible with the nasal mucosa,

Table 1: Solubility of frovatriptan succinate in different oils, surfactants and cosurfactant

\begin{tabular}{|c|c|c|c|c|c|}
\hline Oil & Solubility (mg/ml) & Surfactants & Solubility (mg/ml) & Co-surfactants & Solubility $(\mathrm{mg} / \mathrm{ml})$ \\
\hline Capmul MCM & $39.23 \pm 1.20$ & Cremophor EL & $41.69 \pm 1.15$ & Propylene glycol & $112.86 \pm 1.30$ \\
\hline Isopropyl myristate & $35.33 \pm 1.12$ & Tween 80 & $35.15 \pm 1.31$ & Ethanol & $48.15 \pm 1.12$ \\
\hline Oleic Acid & $22.11 \pm 1.36$ & Cremophor RH-40 & $25.13 \pm 1.52$ & PEG 400 & $23.32 \pm 1.23$ \\
\hline Maisine 35-1 & $13.24 \pm 1.28$ & Labrasol & $15.04 \pm 1.08$ & Plurol olieque & $15.63 \pm 1.12$ \\
\hline Medium chain triglycerides & $5.13 \pm 1.14$ & Labrafil M 2125 CS & $12.34 \pm 1.21$ & - & - \\
\hline
\end{tabular}

Data were expressed as mean \pm S.D., $(n=3)$

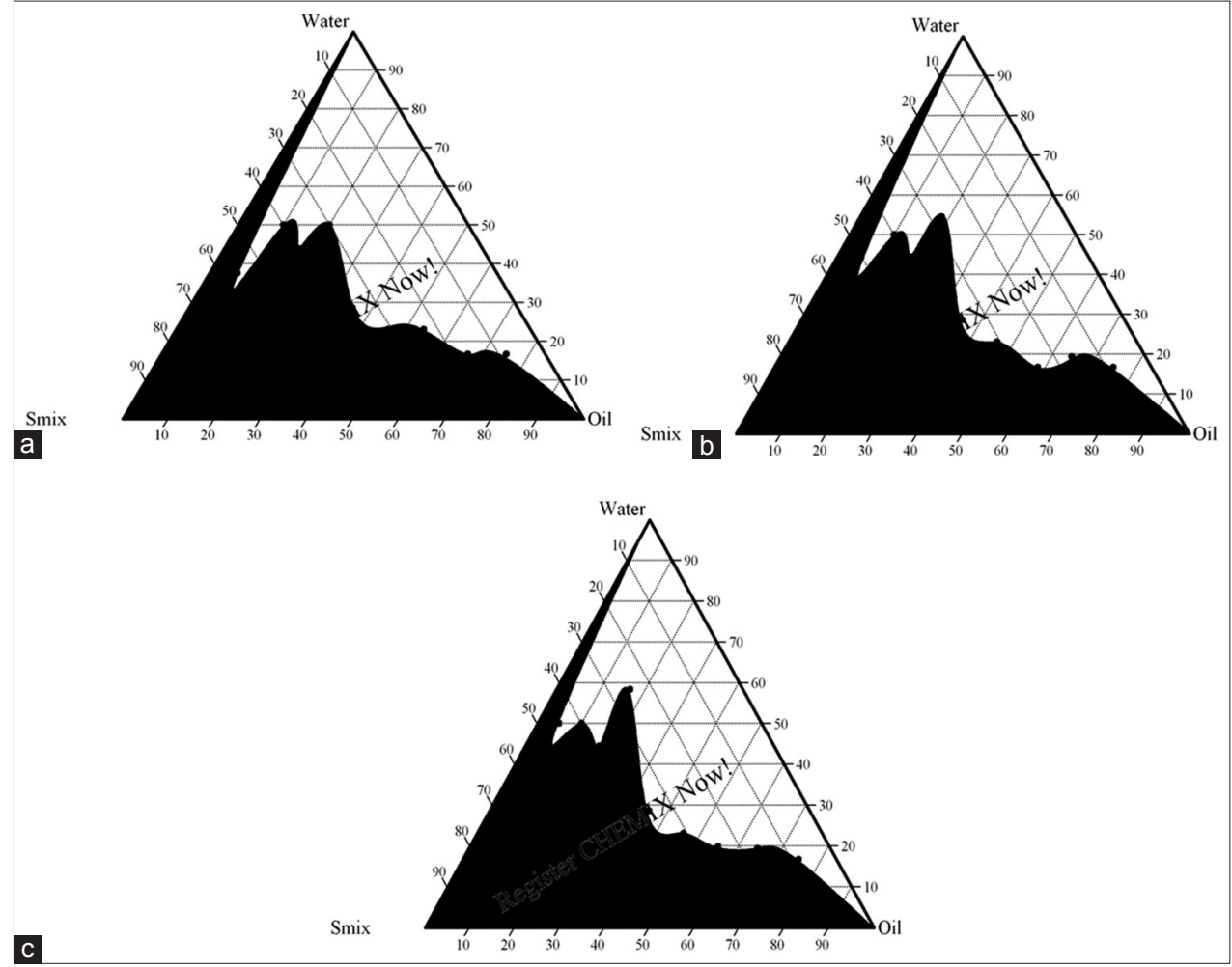

Fig. 1: Pseudoternary phase diagram composed of Capmul MCM (oil), Cremophor EL (surfactant), propylene glycol (cosurfactant), and water at various $S: \operatorname{Cos}\left(S_{\text {mix }}\right)$ ratios, (a) 4:1, (b) 3:1, (c) 2:1 
which falls in nasal pH ranges from 4.5 to 6.5 . Hence, it will not cause any nasal irritation after administration.

\section{Viscosity}

The significance of this study narrates its spreadability/drainage into the nasal cavity after application onto nasal mucosal sites. The viscosity of ME formulation was found low and it could be administered easily in the nasal cavity. Furthermore, low viscosity is suitable to fill ME in a container. Results are shown in Table 3. The rheological behavior of the ME was studied using small volume adaptor.

\section{Refractive index}

The refractive index is a measure of clarity. These MEs were characterized for their transparency to light. The values of the refractive index were nearer to the refractive index of water. This indicates good transparency of the prepared MEs. The refractive index of all MEs is shown in Table 3.

\section{Particle size (globule size of dispersed phase) and zeta potential} Mean globule size of drug loaded all ME formulations are given in Table 3. Formulation F4 showed mean globule size of $457.5 \mathrm{~nm}$ which was maximum, whereas F5 has shown globule size of $142.0 \mathrm{~nm}$ which was least among all formulations. This is shown in Fig. 2. All the formulations, except $\mathrm{F} 4$, were showing globule size within the range of $20-200 \mathrm{~nm}$ which is well accepted for ME. Polydispersity index (PDI) indicates the uniformity of droplet size within the formulation. Higher the polydispersity index, lower the uniformity of the droplet size in the formulation. PDI is the measure of uniformity of particle size in the formulation and $<1$ is desirable [27]. All batches of MEs F1-F7 showed PDI in the range of 0.385-0.431. Thus, more uniformity in droplet size and more thermodynamic stability of formulations was obtained. Measurement of zeta potential was carried out to determine the stability of oil droplets in the ME system. Values of the zeta potential of all ME formulations are shown in Table 3. Practically, the values of the zeta potential of all formulations were found in the range of -17.7 to $-7.8 \mathrm{mV}$. Aggregation of globules was observed due to the negative charge of the droplets. The values of zeta potential indicate that the system is not fully but relatively stable [28]. A negative value indicates that systems have good conductivity, so it also confirms that developed MEs were o/w type. Fig. 3 indicates the value of zeta potential of the optimized ME.

\section{Drug content}

The content of frovatriptan succinate in MEs as given in Table 3 was found to be in the range of $96.57 \pm 1.10$ to $99.19 \pm 1.12 \%$, which confirms almost

Table 2: Simplex lattice design for the formulation of frovatriptan succinate microemulsion

\begin{tabular}{llll}
\hline batch Code & \multicolumn{3}{l}{ Composition of independent variables (\%) } \\
\cline { 2 - 4 } & Oil $\left(\mathbf{X}_{\mathbf{1}}\right)$ & Surfactant $\left(\mathbf{X}_{\mathbf{2}}\right)$ & Co-surfactant $\left.\mathbf{X}_{\mathbf{3}}\right)$ \\
\hline F1 & 15.50 & 17.50 & 5 \\
F2 & 20.00 & 15.00 & 5 \\
F3 & 17.50 & 17.50 & 5 \\
F4 & 20.00 & 15.00 & 5 \\
F5 & 15.00 & 15.00 & 10 \\
F6 & 15.00 & 20.00 & 5 \\
F7 & 17.50 & 15.00 & 7.5 \\
\hline
\end{tabular}

$\mathrm{X}_{1}, \mathrm{X}_{2}$ and $\mathrm{X}_{3}$ are apex points on triangle of simplex lattice design complete solubilisation of drug in formulation resulting transparent solution. Drug-free ME (blank ME) diluted in a similar manner showed no significant peak at $244 \mathrm{~nm}$ indicating an absence of any interference.

\section{In vitro drug diffusion study}

To simulate physiological conditions in the nasal cavity, phosphate buffer 6.4 and temperature $34^{\circ} \mathrm{C}$ were selected. Cumulative per cent drug release through dialysis membrane of different ME formulations was found to be in the range of $65-85 \%$ at the end of $7 \mathrm{~h}$. Formulation $\mathrm{F} 5$ has shown maximum drug release in $7 \mathrm{~h}$ (Fig. 4). Initially, for all formulations, drug release rate was faster due to release of drug present in the aqueous phase which is a continuous phase in ME and later on decreased may be due to drug release from oil droplets. Correlation coefficient, $\mathrm{R}^{2}$ equal to 0.9917 and 0.9909 , showed first orders for F2 and F5 formulations, respectively. Formulation F6 has shown slow drug release and even 85\% could not obtain in $7 \mathrm{~h}$. This may due to the high viscosity of formulation as compared to others and probably due to thick gel-like structure formation due to the very high amount of surfactant used.

\section{Ex vivo permeation study}

As formulation F5 exhibited good in vitro drug release profile with favorable evaluation parameters, the formulation F5 was chosen to observe the permeation of drug through sheep nasal mucosa. The optimized ME (F5) was studied ex vivo for drug release from the sheep nasal mucosa for $7 \mathrm{~h}$ [29]. Permeation of the drug from optimized formulation, F5, was $82.32 \%$ and followed the Peppas model at the end of $7 \mathrm{~h}$. Percentage drug release and average flux after $7 \mathrm{~h}$ obtained $82.32 \%$ and $9.51 \mathrm{mcg} / \mathrm{cm}^{2} / \mathrm{min}$, respectively. Thus, ME system composed of frovatriptan succinate is a promising approach for targeting drug in brain tissues.

\section{Histopathological study}

One of the requirements of formulation development is that formulation should not produce any nasal mucosal irritation [26]. The excipients were selected on the basis of "generally regarded as safe material." This histopathology study helps to evaluate toxicity of ME using sheep nasal mucosa $[30,31]$. We can hypothesize the possible toxicity of said formulation to the human nasal mucosa. For better differentiation and confirmation, positive and negative controls were used to compare the effect of optimized ME (F5) on sheep nasal mucosa. The images of histopathology study are shown in Fig. 5.

From Fig. 5, the cellular structure of the nasal mucosa was retained after the treatment of the optimized ME formulation and phosphate buffer $\mathrm{pH} 6.4$ and it is damaged with the positive control of IPA. It is seen under the microscope that original structure was not retained after treatment with IPA. Thus, there was no toxicity produced by the optimized ME. Hence, it can be concluded that the optimized ME was safe.

Mucoadhesive strength of the optimized ME formulation was carried out. The mucoadhesive strength of the tested formulation was found $328.85 \pm 2.00$ dyne $/ \mathrm{cm}^{2}$.

Optimization using simplex lattice design

For the development of a ME system, various compositions of $\mathrm{S}_{\text {mix }}$ were used. During trial batches, it was found that $\mathrm{S}_{\text {mix }}$ compositions were

Table 3: Evaluation parameter of microemulsion formulations F1-F7

\begin{tabular}{lllllll}
\hline Batch code & $\mathbf{p H}$ & Viscosity (cps) & Refractive index & Drug content (\%) & Particle size (nm) & Zeta potential (mv) \\
\hline F1 & $5.2 \pm 0.1$ & $96 \pm 1.09$ & $1.39 \pm 0.11$ & $98.13 \pm 1.00$ & 155.4 & -10.6 \\
F2 & $5.2 \pm 0.1$ & $81 \pm 1.28$ & $1.36 \pm 0.18$ & $97.42 \pm 1.11$ & 143.5 & -17.7 \\
F3 & $5.3 \pm 0.1$ & $116 \pm 1.32$ & $1.38 \pm 0.17$ & $99.19 \pm 1.12$ & 160.5 & -13.4 \\
F4 & $4.7 \pm 0.1$ & $103 \pm 1.50$ & $1.36 \pm 0.12$ & $98.64 \pm 1.20$ & 457.5 & -9.28 \\
F5 & $5.4 \pm 0.1$ & $101 \pm 1.43$ & $1.37 \pm 0.21$ & $98.25 \pm 1.10$ & 142.0 & -10.4 \\
F6 & $5.3 \pm 0.1$ & $113 \pm 1.27$ & $1.35 \pm 0.21$ & $96.57 \pm 1.10$ & 173.1 & -8.35 \\
F7 & $4.9 \pm 0.1$ & $97 \pm 1.28$ & $1.36 \pm 0.12$ & $97.88 \pm 1.13$ & 239.4 & \\
\hline
\end{tabular}

Data were expressed as mean \pm S.D., $(n=3)$ 


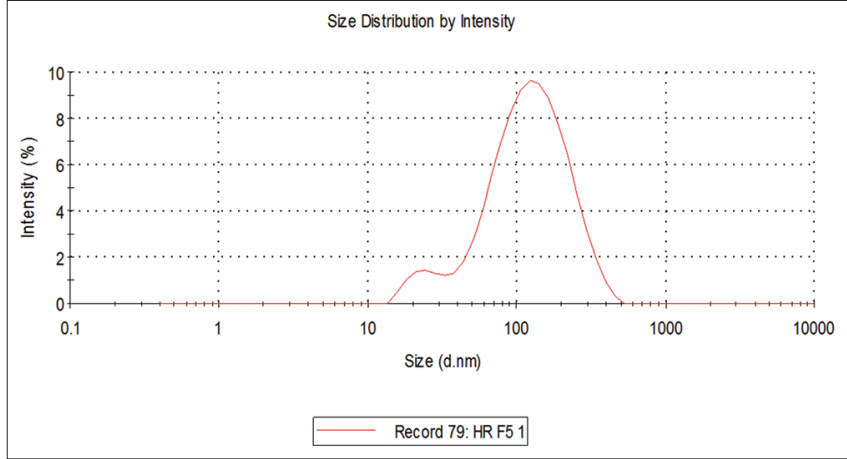

Fig. 2: Average globule size of the optimized microemulsion formulation

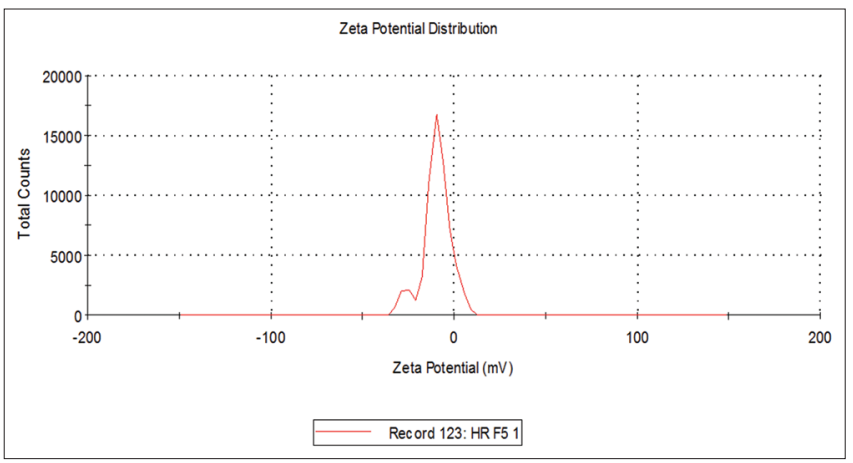

Fig. 3: Zeta potential of the optimized microemulsion formulation

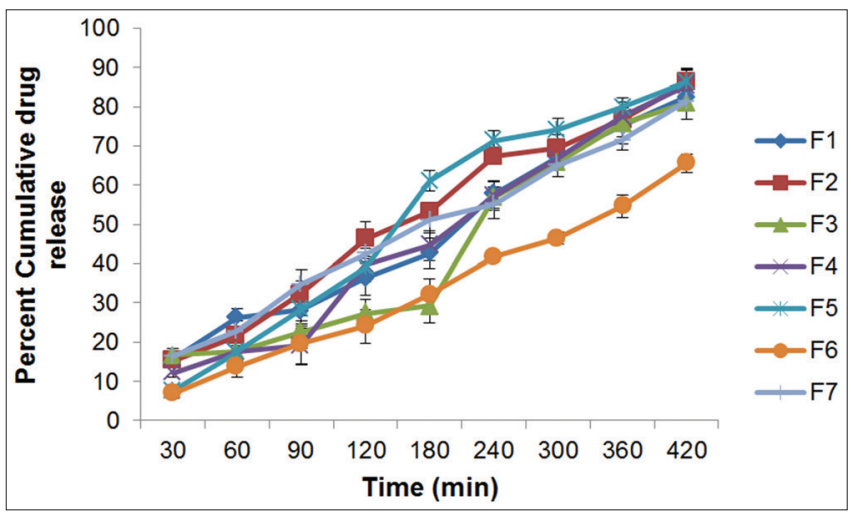

Fig. 4: Drug release of nasal microemulsion formulations

important parameters which may affect the drug release from the formulation. The factorial design was applied and carried out with the aim of finding the optimum concentration of oil, surfactant, and cosurfactant level to achieve a drug release above $85 \%$ from ME formulation. Simplex lattice design was helpful to evaluate the formulation of threecomponent system. The total of mixture component was $100 \%$. The percentage of drug release after $7 \mathrm{~h}$ was ranged from $65.63 \%$ to $86.33 \%$ in F1 to F7 ME formulations. The polynomial equations can be used to draw conclusions after considering the magnitude of the coefficient and the mathematical sign it carries, i.e., positive or negative.

The equation for drug release is as follows:

$$
\begin{aligned}
\text { Ydrug release }= & 80.63+0.41 \mathrm{X}_{1}-12.35 \mathrm{X}_{2}-2.89 \mathrm{X}_{3}+0.76 \mathrm{X}_{1} \mathrm{X}_{2}- \\
& 0.48 \mathrm{X}_{1} \mathrm{X}_{3}+1.27 \mathrm{X}_{2} \mathrm{X}_{3}+1.01 \mathrm{X}_{1} \mathrm{X}_{2} \mathrm{X}_{3}
\end{aligned}
$$

To evaluate the percentage drug release equation (1), different models were selected. $\mathrm{F}$ value and $\mathrm{p}$ value imply that linear model was

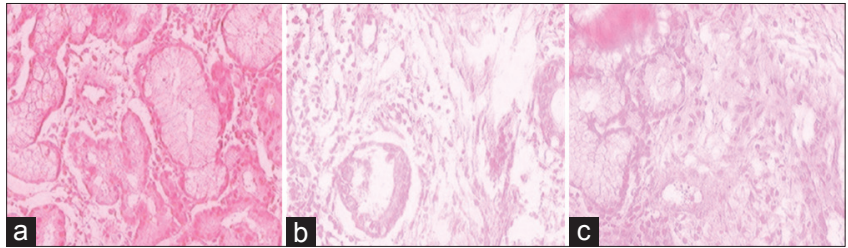

Fig. 5: Histopathological evaluation of sheep nasal mucosa: (a) pH 6.4 negative control, (b) Isopropyl alcohol positive control, (c) optimized microemulsion formulation

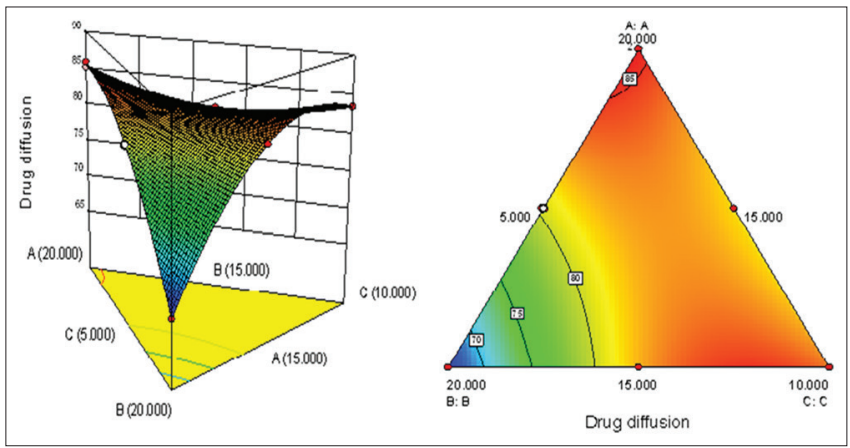

Fig. 6: Response surface and contour plot for the effect of independent variable on drug release

significant as shown in Table 4. $X_{1}$ shows the agonistic effect to drug release. $X_{2}$ and $X_{3}$ show antagonistic effect. A common effect of $X_{2} X_{3}$ and $\mathrm{X}_{1} \mathrm{X}_{2} \mathrm{X}_{3}$ shows additive effect, while $\mathrm{X}_{1} \mathrm{X}_{3}$ shows negative effect. Hence, linear interaction increases drug release of the ME. Factor $\mathrm{X}_{2} \mathrm{X}_{3}$ has more significant effect on drug release through the dialysis membrane than other coefficients, thus necessary for drug release.

\section{Response surface analysis}

The linear models generated by regression analysis were further used to construct three-dimensional response plots, in which response parameter $Y$ was represented by a curvature surface as a function of X. Fig. 6 shows the effect of three factors on percentage drug release at the end of $7 \mathrm{~h}$ and also shows a linear relationship between the three independent variables, i.e., $X_{1}, X_{2}$, and $X_{3}$ on response $Y$ (percentage drug release).

An optimization technique using the desirability approach was employed to develop a new formulation with the desired response. The new formulation was validated for percentage drug release. No significant difference was observed in experimental and predicted values of percentage drug release as shown in Table 5.

\section{Animal study}

Analytical procedure and sample preparation

Under chromatographic conditions, retention time was $2.08 \mathrm{~min}$ for frovatriptan succinate and $2.18 \mathrm{~min}$ for internal standard metoclopramide hydrochloride. A $0.1 \%$ formic acid was added in the mobile phase to generate proton ions. ESI was performed using positive ion mode, and MRM fragmentation reaction was carried out for each drug. Quantification of protonated molecules of frovatriptan succinate at $\mathrm{m} / \mathrm{z} 213.1$ was occurred and m/z 227.2 for internal standard metoclopramide hydrochloride. Parent ion of frovatriptan succinate was determined at $\mathrm{m} / \mathrm{z} 244.5$, as the molecular weight of drug is 243.5 (Fig. 7). Thus, it proves characteristic property of drug. Detection was performed using an API-4000 QTRAP instrument (Applied Biosciences ABsciex). A calibration curve was constructed in the range of $10-800 \mathrm{ng} / \mathrm{ml}$. A mean correlation coefficient $\left(\mathrm{R}^{2}\right)$ for the calibration curve was 0.9985 . The accuracy for frovatriptan succinate was found to be in acceptable range ( -15 to $15 \%)$. 
Table 4: Summary of ANOVA for dependent variables from simplex lattice design

\begin{tabular}{llllll}
\hline Response & Sum of squares & Df & Mean square & F value & p value \\
Percentage drug release & 285.65 & 5 & 57.13 & 254.53 & 0.0475 significant \\
\hline $\mathrm{p}<0.05$ & & &
\end{tabular}

Table 5: Validation of optimized batch F5

\begin{tabular}{lllll}
\hline S. No. & Parameter & Experimental value & Predicted value & Percentage error \\
1 & Percentage drug release & $86.33 \%$ & $84.88 \%$ & +1.67 \\
\hline
\end{tabular}

Percent error not significant; tested for optimized formulation

Table 6: Pharmacokinetic parameters of frovatriptan succinate-loaded microemulsion for oral and nasal route

\begin{tabular}{|c|c|c|c|}
\hline Pharmacokinetic parameters & Route of administration & Plasma & Brain tissues \\
\hline \multirow{2}{*}{$\mathrm{T}_{\max }(\mathrm{h})$} & Nasal & $2 \pm 0.00$ & $2 \pm 0.00$ \\
\hline & Oral & $2 \pm 0.00$ & $2 \pm 0.00$ \\
\hline \multirow{2}{*}{$\mathrm{C}_{\max }(\mathrm{ng} / \mathrm{ml})$} & Nasal & $331 \pm 3.72$ & $181 \pm 1.51$ \\
\hline & Oral & $161 \pm 4.22$ & $52 \pm 1.08$ \\
\hline \multirow[t]{2}{*}{$\mathrm{AUC}_{0-6}$ (ng.h/ml) } & Nasal & $926.1 \pm 4.71$ & $390.0 \pm 2.08$ \\
\hline & Oral & $581.5 \pm 2.42$ & $118.6 \pm 2.70$ \\
\hline \multirow{2}{*}{$\mathrm{AUC}_{\text {brain tissue }} / \mathrm{AUC}_{\text {plasma }}(\%)$} & i) Nasal & 42.11 & \\
\hline & ii) Oral & 20.39 & \\
\hline DTI & --- & 2.06 & \\
\hline
\end{tabular}

Data were expressed as mean \pm S.D., (n=3), DTI: Drug targeting index

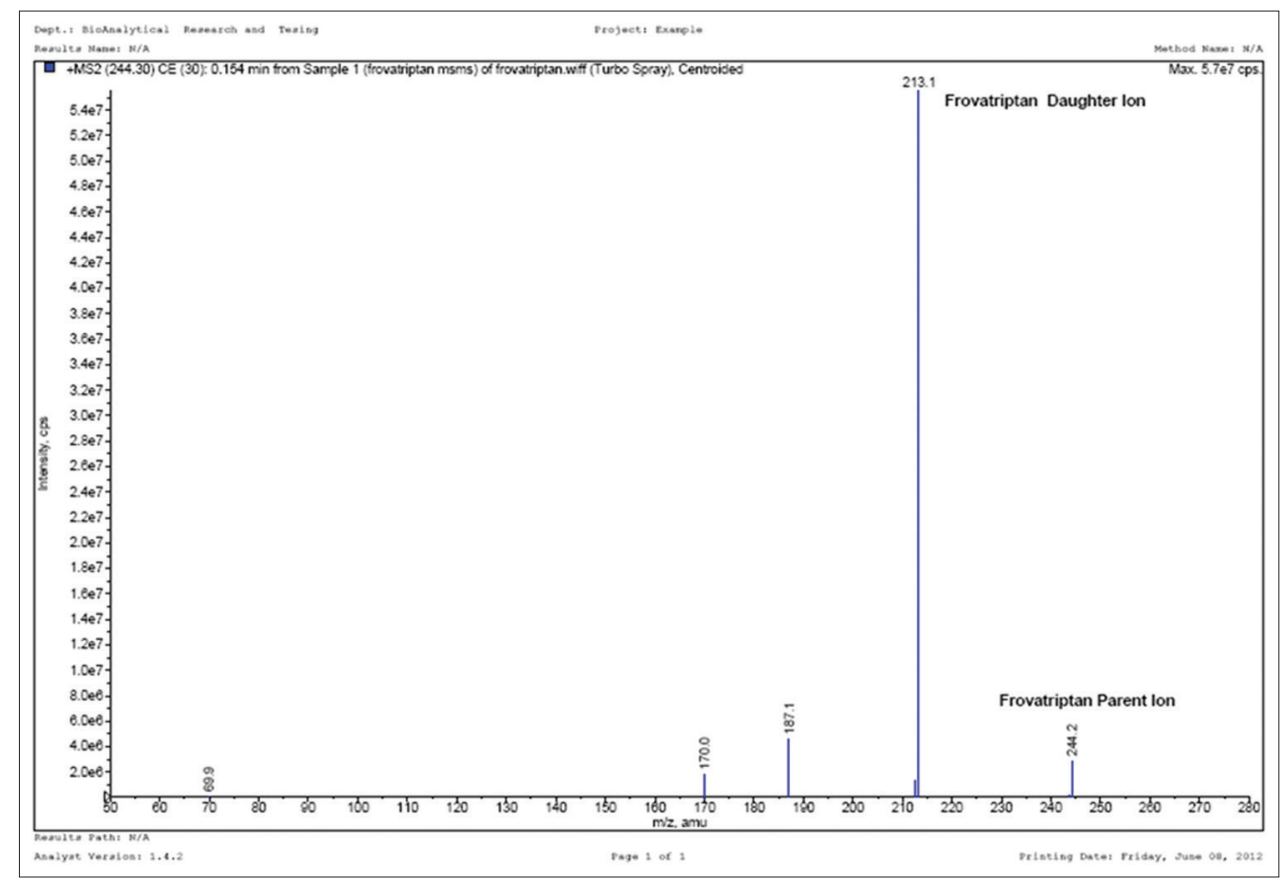

Fig. 7: MS tune of frovatriptan succinate

Pharmacokinetics: Estimation of concentrations of drug in plasma Pharmacokinetic parameters [32,33] were calculated from plasma concentration-time profiles and are shown in Fig. 8. Values of $\mathrm{C}_{\max } \mathrm{T}_{\text {max }}$ $\mathrm{AUC}_{0-6}(\mathrm{ng} . \mathrm{h} / \mathrm{ml})$, and ratio of $\left(\mathrm{AUC}_{\text {brain tissue }} / \mathrm{AUC}_{\text {plasma }}\right) \%$ are shown in Table 6 . The $\mathrm{C}_{\max }$ attained by frovatriptan in plasma by nasal ME was 2 times greater than the oral solution. The $\mathrm{AUC}_{0-6}$ attained by nasal $\mathrm{ME}$ in plasma was 1.59 times greater than the oral solution. It was observed that $\mathrm{C}_{\max }$ was achieved higher in plasma by nasal ME compared to the oral aqueous solution at fixed time point.

\section{Estimation of concentrations of drug in brain tissues}

The $\mathrm{C}_{\text {max }}$ attained by frovatriptan in the brain tissues by nasal ME was 3.5 times greater than the oral solution. The $\mathrm{AUC}_{0-6}$ attained by nasal ME was 3.29 times greater than the oral solution. It was observed that $\mathrm{C}_{\max }$ was achieved higher in nasal ME compared to the oral aqueous solution at fixed time point.

Table 6 shows the concentration of frovatriptan succinate in the brain tissues following intranasal and oral administration. This is shown in Fig. 9. The ratio of $\left(\mathrm{AUC}_{\text {brain tissue }} / \mathrm{AUC}\right.$ plasma $) \%$ for nasal route and oral route was 42.11 and 20.39, respectively. Thus, brain-blood ratios of frovatriptan succinate were found to be higher for formulation administered nasally. This confirms direct nose to brain transport. The concentrations of frovatriptan succinate in the brain following intranasal administration of ME was found to be significantly higher at all sampling points compared to oral administration for $6 \mathrm{~h}$. Therefore, it can be concluded that concentration of drug in brain tissue could be increase successfully by nasal route rather than the 


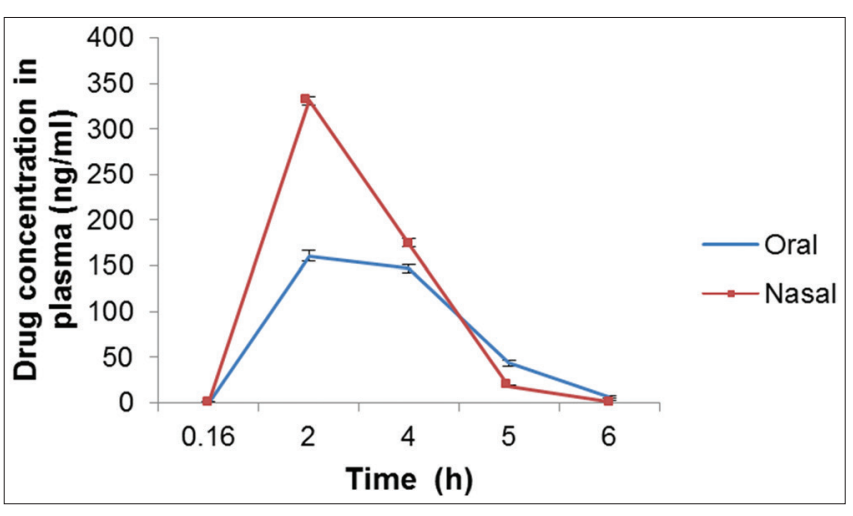

Fig. 8: Drug concentrations in plasma after nasal and oral dose in rat

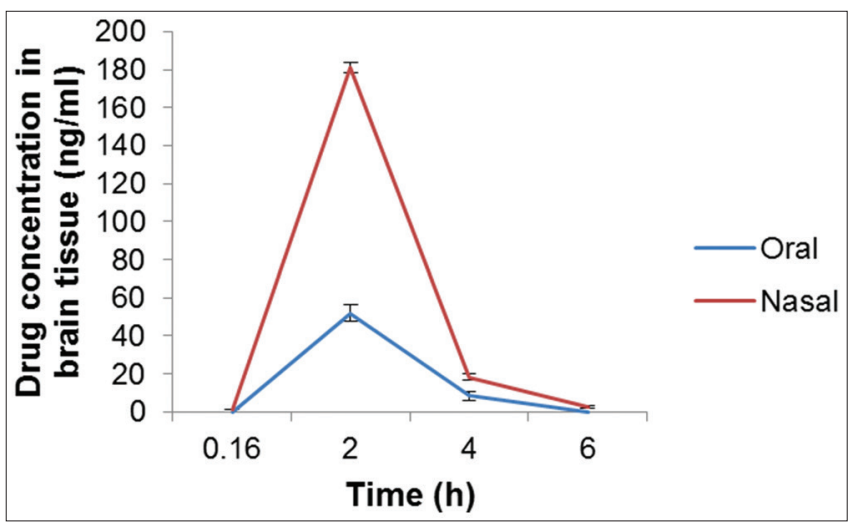

Fig. 9: Drug concentrations in brain tissue after nasal and oral dose in rat

oral route [15]. Amount of frovatriptan succinate in brain tissue after nasal administration is attributed to two pathways through systemic pathway and olfactory pathway; however, the latter is major [34]. Jaiswal et al. described mechanism of brain targeted intranasal delivery via olfactory epithelium [35].

\section{Estimation of drug targeting}

The DTI for brain tissue was calculated [33]. DTI represents a time average partitioning ratio. DTI 2.06 was found satisfactory. It indicates that nasal route is an alternative pathway for frovatriptan succinate acting on brain tissues through the olfactory pathways. From pharmacokinetic studies, it can be concluded that nasal route provides a passage for frovatriptan succinate to reach into brain tissues. The component of optimized ME was suitable for frovatriptan succinate for permeation across the nasal mucosa through olfactory pathways.

Stability study was carried out in the humidity chamber (Oswal Scientific) where the temperature was set at $40 \pm 2^{\circ} \mathrm{C} / 75 \pm 5 \%$ relative humidity for 3 months. At every 2 weeks, samples were withdrawn and analyzed for physicochemical parameters. At the end of $3^{\text {rd }}$ month, the comparative study was tabulated and any change in macroscopic appearance such as transparency, homogeneity, drug precipitation, turbidity, $\mathrm{pH}$, refractive index, drug content \%, and drug released was evaluated. There was no significant difference between the initial and respective values during and at the end of the study.

\section{CONCLUSION}

To conclude, the ME of frovatriptan succinate was successfully prepared by the ME of frovatriptan succinate was successfully prepared by water titration method and demonstrated in Swiss albino rats to deliver frovatriptan succinate in effectively to the brain following intranasal administration. This study demonstrated the effectiveness and feasibility of intranasal delivery of frovatriptan succinate through ME. Further clinical data are needed to produce benefit-to-risk ratio for suitability in clinical study and practice.

\section{ACKNOWLEDGMENT}

The authors are thankful to Glenmark Generic Ltd., Navi Mumbai, India, for gift sample of frovatriptan succinate.

\section{AUTHORS' CONTRIBUTION}

Upendra C Galgatte, Pravin D Chaudhari, contributed to this manuscript to justify authorship criteria.

\section{CONFLICTS OF INTEREST}

Authors report no conflicts of interest.

\section{REFERENCES}

1. Arora P, Sharma S, Garg S. Permeability issues in nasal drug delivery. Drug Discov Today 2002;7:967-75.

2. Dhuria SV, Hanson LR, Frey WH $2^{\text {nd }}$. Intranasal delivery to the central nervous system: Mechanisms and experimental considerations. J Pharm Sci 2010;99:1654-73.

3. Chen YW, Su KS, Chang SF. editors. Anatomy and physiology of the nose. In: Nasal Systemic Drug Delivery: Drugs and the Pharmaceutical Sciences. New York: Marcel Dekker Inc.; 1989. p. 1-19.

4. Illum L. Transport of drugs from the nasal cavity to the central nervous system. Eur J Pharm Sci 2000;11:1-8.

5. Talegaonkar S, Mishra PR. Intranasal delivery: An approach to bypass the blood brain barrier. Indian J Pharmacol 2004;36:140-7.

6. Ugwoke MI, Agu RU, Verbeke N, Kinget R. Nasal mucoadhesive drug delivery: Background, applications, trends and future perspectives. Adv Drug Deliv Rev 2005;57:1640-65.

7. Block LH. Pharmaceutical emulsions and microemulsions. In: Liberman HA, Rieger MM, editors. Pharmaceutical Dosage Forms:

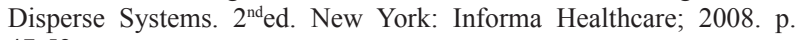
47-52.

8. Bölcskei H, Farkas B, Kocsis P, Tarnawa I. Recent advancements in anti-migraine drug research: Focus on attempts to decrease neuronal hyperexcitability. Recent Pat CNS Drug Discov 2009;4:14-36.

9. Kelman L. Review of frovatriptan in the treatment of migraine. Neuropsychiatr Dis Treat 2008;4:49-54.

10. Balbisi EA. Frovatriptan: A review of pharmacology, pharmacokinetics and clinical potential in the treatment of menstrual migraine. Ther Clin Risk Manag 2006;2:303-8.

11. Frovatriptan Monograph. Available from: http:/www.drugs.com/ frovatriptansuccinate.html. [Last accessed on 2018 Jul 31].

12. Lianli L, Nandi I, Kim KH. Development of an ethyl laurate-based microemulsion forrapid-onset intranasal delivery of diazepam. Int $\mathrm{J}$ Pharm 2002;237:77-85.

13. Yadav SR, Gholse YN, Kawtikwar PS, Sakarkar DM. Design and evaluation of almond oil based water-in-oil mucoadhesive microemulsionfor nose to brain delivery. Int J Pharm Res 2011;3:48-52.

14. Vyas TK, Babbar AK, Sharma RK, Singh S, Misra A. Intranasal mucoadhesive microemulsions of clonazepam: Preliminary studies on brain targeting. J Pharm Sci 2006;95:570-80.

15. Vyas TK, Babbar AK, Sharma RK, Misra A. Intranasal mucoadhesive microemulsions of zolmitriptan: Preliminary studies on brain-targeting. J Drug Target 2005;13:317-24.

16. Singh G, Rawat N, Singh K, Sarwal A, Sinha VR. Investigating the potential of an antidepressant intranasal mucoadhesive microemulsion. Int J Pharm Pharm Sci 2018;10:125-32.

17. Kawatikwar PS, Kulkarni NP, Yadav S, Sakarkar DM. Formulation and evaluation of an anti-epileptic drug loaded microemulsion for nose to brain delivery. Asian J Pharm 2009;3:143-7.

18. Pathak R, Dash RP, Misra M, Nivsarkar M. Role of mucoadhesive polymers in enhancing delivery of nimodipine microemulsion to brain via intranasal route. Acta Pharm Sin B 2014;4:151-60.

19. Zhu W, Yu A, Wang W, Dong R, Wu J, Zhai G, et al. Formulation design of microemulsion for dermal delivery of penciclovir. Int J Pharm 2008; 360:184-90

20. Carter SJ, editor. Dispensing for Pharmaceutical Students. $12^{\text {th }}$ ed. New York: CBS Publishers and Distributors Pvt., Ltd.; 2008. p. 120-2.

21. Dwivedi N, Kurmi BD, Kesharwani P, Shah J. Generation dependent 
targeting potential of donepezil loaded poly (propyleneimine) dendrimer through goat nasal mucosa. Int J Pharm Pharm Sci 2018;10:80-7.

22. Pote CW. Development Optimization and Evaluation of Microemulsion for Nasal Delivery [Dissertation]. Pune University; 2012.

23. Bhanushali RS, Gatne MM, Gaikwad RV, Bajaj AN, Morde MA. Nanoemulsion based intranasal delivery of antimigrain drugs for nose to brain targeting. Indian J Pharm Sci 2009;71:707-9.

24. Choi HG, Oh YK, Kim CK. In situ gelling and mucoadhesive liquid suppository containing acetaminophen: Enhanced bioavailability. Int J Pharm 1998;165:23-32.

25. Laugher L, Briggs R, Doughty J, Noctor TA. Development of an analytical methodology from toxicokinetic to clinical studies for the anti-migraine drug frovatriptan. Chromatographia 2000;52:S113-9.

26. Zhang Q, Jiang X, Jiang W, Lu W, Su L, Shi Z, et al. Preparation of nimodipine-loaded microemulsion for intranasal delivery and evaluation on the targeting efficiency to the brain. Int J Pharm 2004;275:85-96.

27. Patel ZB, Patel KS, Shah AS, Surti NI. Preparation and optimization of microemulsion of rosuvastatin calcium. J Pharm Bioallied Sci 2012; 4:S118-9.

28. Bhattacharjee S. DLS and zeta potential - What they are and what they are not? J Control Release 2016;235:337-51.

29. Kumar A, Sharma P, Chaturvedi A, Jaiswal D, Bajpai M,
Choudhary M, etal.Formulation development of sertralinehydrochloride microemulsion for intranasal delivery. Int J Chem Tech Res 2009;1: 941-7.

30. Piao HM, Balakrishnan P, Cho HJ, Kim H, Kim YS, Chung SJ, et al. Preparation and evaluation of fexofenadine microemulsion for intranasal delivery. Int J Pharm 2010;309-16.

31. Mandal S, Mandal SS, Naazneen S, Patel VB, Mandal PA. Design and development of saquinavir microemulsion for the oral bioavailability enhancement. Int J Pharm Tech Res 2009;4:1442-8.

32. Botner S, Sintov AC. Intranasal delivery of two benzodiazepines, midazolam and diazepam by a microemulsion system. Pharmacol Pharm 2011;2:180-8

33. Lu W, Jiang W, Chen J, Yin M, Wang Z, Jiang X, et al. Modulation of brain delivery and copulation by intranasal apomorphine hydrochloride. Int J Pharm 2008;349:196-205.

34. Kumar M, Misra A, Babbar AK, Mishra AK, Mishra P, Pathak K. Intranasal nanoemulsion based brain targeting drug delivery system of risperidone. Int J Pharm 2008;358:285-91.

35. Jaiswal PL, Darekar AB, Saudagar RB. A recent review on nasal microemulsion for treatment of CNS disorder. Int J Curr Pharm Res 2017;9:5-13. 